\title{
Editorial
}

\section{The Emerging Trends in Dental Practices and Need of Improved Knowledge Dissemination}

\author{
Varun Jindal ${ }^{1}$ Deepti Jindal ${ }^{2}$ \\ ${ }^{1}$ Department of Conservative Dentistry and Endodontics, Bhojia \\ Dental College and Hospital, Solan, Himachal Pradesh, India \\ 2Department of Oral and Maxillofacial Pathology, Bhojia Dental \\ College and Hospital, Solan, Himachal Pradesh, India
}

Dentistry as a field of medicine is uniquely significant in the day-to-day life of people across all age groups. Dentists across the world treat and serve millions of patients every day suffering from several dental ailments ranging from dental cavities, tooth dislocation, enamel-related issues to dental extractions. Despite the field contributing toward the wellness and quality of life for patients from different age groups, the specialty still needs a lot of contribution from practicing clinicians, students, and researchers for quality clinical publications as compared with other subject areas. Factors including lack of funding in dental research in several developing countries, lack of interuniversity and interstate collaboration between dental institutes and hospitals, and mushrooming of several dental colleges and institutes significantly add toward the same.

In addition to the above, existence of several dental journals with overlapping area of interest just contribute toward dilution of the very significance of focused dental publications. Moreover, in India, students, residents, and even practicing clinicians are deeply influenced by regulatory guidelines where it is mandatory to publish articles in journals recognized by leading indexing and abstracting portals. In doing so, the regulatory agencies fail to understand that they are pushing enthusiastic universities/societies and respective university/society-run dental journals into a vicious cycle. Leading abstracting and indexing portals are not there to help journals grow and gain good submissions, rather they would list journals that are having interesting and significant clinical content.

For a new journal, it is a huge struggle to publish strong clinical content until the time it is listed in major abstracting and indexing portals, where the students, residents, and clinicians would always be reluctant to submit to a journal not listed in the major bibliographic portals.

The only way left to the Editors of these journals is to solicit content until the time the listing happens. To encounter such situations, it is the need of the hour that members of the universities and societies to which the concerned journal is affiliated should actively contribute toward the content, until the journal gets its due listing.

Dental Journal of Advance Studies (DJAS) is a journal that is multidisciplinary in nature, and its very existence is to ensure that dissemination of clinical information related to dental disciplines is well taken care of to the wide group of students and clinicians. The journal is a unique platform provided by Bhojia Dental Hospital and Himachal Pradesh University that are committed toward spreading and exchanging information among students and practitioners in the field. The journal aims to serve as a platform for young investigators working in hospitals and individual practicing clinicians from the remotest areas to come forward and share their knowledge with the world. Having partnered with Thieme Medical Publishers, DJAS aims to take investigations from the farthest corners of India, rural, suburban, and urban areas, to the global level and hope to serve as a medium for collaborations among individual practitioners and dental groups from institutes and hospitals.

In summation, we emphasize that the university and the journal aim at disseminating quality information for the readers covering all specialties in dental practice and research. The same can only be accomplished when potential authors come forward and contribute toward the same. We therefore would encourage potential authors to submit your content at www.manuscriptmanager.net/djas. The journal can be read at www.thieme.com/djas.

\footnotetext{
Address for correspondence

Varun Jindal, BDS, MDS, Department

of Conservative Dentistry and

Endodontics, Bhojia Dental College

and Hospital, Baddi 173205 ,

Solan, Himachal Pradesh, India

(e-mail: editordjas@gmail.com).
}

DOI https://doi.org/

$10.1055 / \mathrm{s}-0039-1677776$

ISSN 2321-1482.
C 2018 Bhojia Dental College and Hospital affiliated to Himachal Pradesh University
License terms

$($ (1) $\Theta \circledast$ 
